Robert M L Baker, Jr. (2010),

"Applications of High-Frequency Gravitational Waves to the Global War on Terror"

After peer review accepted for Publication in the Proceedings of the Space, Propulsion and Energy Sciences International Forum (SPESIF 2010), February 23-26, John Hopkins University Applied Physics Laboratory, Laurel, MD, U.S.A., Edited by Glen Robertson. (Paper 001), American Institute of Physics Conference Proceedings, Melville. NY, USA Volume 1208.

The paper is presented after the following peer review: 


\section{Referee Correspondence}

\section{RESPONSE TO REFEREE COMMENTS ON PAPER 001 (September 9, 2009):}

Some of the statements in the abstract are self-contradictory; it is stated that because of "their ability to pass through all material without attenuation, HFGWs could be utilized for uninterruptible, very lowprobability-of-intercept (LPI), high-bandwidth communications" which is clearly contradictory since the HFGWs must interact with a receiver to be useful.

I attempted to clarify this in the attached manuscript as follows:

"Although HFGWs do not interact with and are not absorbed by ordinary matter, their presence can be detected by their distortion of spacetime as measured for low-frequency gravitational waves by the Laser Interferometer Gravitational Observatory or LIGO (Abbott, et al., 2008), Virgo (Ballardio, et al, 2001), GEO600 (Hogan, 2008) and for HFGWs by detection photons generated from electromagnetic beams having the same frequency, direction and phase as the HFGWs in a superimposed magnetic field, the LiBaker HFGW Detector (Baker, Stephenson and Li, 2008; Li et al., 2008; Li et al. 2009), by the change in polarization they produce in a microwave-guide loop as in the Birmingham University Detector (Cruise and Ingley, 2005) and by other such HFGW detection instruments (Chincarini and Gemme, 2003; Nishizawa et al. 2008). None of these effects utilized for detection represent interaction with matter in a way that causes GW absorption, but rather interaction with fields and the detection devices do not attenuate the GWs." The detection instruments measure change in GW polarization, distortion of the fabric of spacetime, change in EM that has the same frequency, phase and direction as the GWs, etc. - none of which attenuates the GW.

Also, I believe HFGW propulsion is certainly very unlikely, and surveillance also is a non starter if one believes the very small cross-section for interaction with ordinary matter.

Too many concepts in the history of Science have been ruled out prior to actual experiments. Conventional propulsion applications of GW have been discussed theoretically by Bonnor and Piper and by Davis and from an astrophysical viewpoint by Bekenstein. The most compelling discussion is found in Landau and Lifshitz where they note qualitatively the possibly significant influence of high-frequency gravitational waves on otherwise static gravitational fields -- certainly a possibility that calls for experimental study. Surveillance applications would depend upon the so-far unknown influence of intervening matter between the HFGW generator and detector in a laboratory experiment (no absorption though) and represents a motivation for such an experiment as stated in the manuscript. The following quotes from the manuscript amplify this idea:

"These important potential HFGW applications are motivations for HFGW research and development and such an R\&D program is recommended for immediate initiation."

"The plausibility of the theoretical applications cannot, however, be adequately determined until after a proof-of-concept test is successfully completed."

"A quantitative analysis must necessarily await a laboratory HFGW generation-detection experiment in order to determine the value of parameters involved."

"Tests with $10^{9} \mathrm{~Hz}$ or higher gravitational waves must be accomplished before the application is either discarded or accepted."

And so on.

Re "Published misinformation" - a citation(s) is needed, and the wording needs to be more neutral. 
Good criticism, therefore I removed the comment. No need to be defensive.

Table 1 of Woods \& Baker gives the total generated power as 4.62E-16W, this is not per molecule pair as stated in the present MS. Reference to "X-rays" under "Surveilance" (sic) is unfortunate since it is an incorrect term, and should be replaced by "rays" or "waves".

Again good points. I removed the utilization of the IR-ring HFGW generation means since it is far too theoretical. I replaced it by a piezoelectric generator first proposed by Dehnen and Romero that can be built from off-the-shelf components.

The quoted sensitivity of HFGW detectors to polarization angle changes (1E-40rad) is surprising and needs a reference or other justification. It seems more likely that interspersed materials will produce a propagation phase change rather than polarization angle change, but even so such a sensitivity to a phase change would also be unprecedented which is why a reference is needed.

I reference Misner, Thorne and Wheeler - not to show specific quantitative changes in HFGWs due to intervening material (no absorption though), but as a motivation for experiment since without experimental evidence at these high frequencies no quantitative information can be obtained by theory.

Regarding propulsion using HFGW, this idea has been around for some time but estimates of its magnitude are tiny. Either an order-of-magnitude calculation needs to be done, or else an explanation of why it doesn't need to be done.

Order of magnitude studies of "conventional" GW propulsion have been accomplished by Bonnor and Piper and by Davis and others and are referenced. The referee is correct that they produce a very small thrust like a "photon rocket" often proposed for interstellar travel. As already noted, the comments by Landau and Lifshitz are more compelling and cannot be evaluated properly prior to the HFGW generationdetection experiment. The parameters for the computer program would come from such a HFGW experiments and are only guestimates at this time.

No need to include a complete computer program (particularly when it includes questionable programming techniques such as $\mathrm{e}^{\wedge} 2$ which on many systems is *considerably* slower and less accurate than $\mathrm{e}^{*} \mathrm{e}$ ); instead, state the equations or principles on which it operates, and any competent scientist/engineer can translate that to whatever his favorite computer language is.

The computer program was included specifically to provide the "order of magnitude calculations" suggested by the referee as a valuable tool for the analyst. Most of the program involves conventional celestial mechanics Kepler-orbit equations (specifically referred to in the Herrick reference), but without considerable background in this field it would be difficult to program these classical equations so I did it for the reader. The TrueBasic high-level language allows "competent scientist/engineer can translate that to whatever his favorite computer language is ..." The program runs on my laptop in a fraction of a second so the difference between the square of the eccentricity and the product and any lost accuracy is trivial.

This MS lacks a Conclusions section.

Done.

Please advise me of any further corrections.

R. Baker

Dr. Baker: the reviewer had these comments to your revisions:

This is much improved and I thank the author for attending to the details of this potentially fine review of the subject. 
All the quantitative estimates I have seen (e.g. Jason report) are so small that it seems difficult to know how to get anything useful from this effect. In the absence of any other quantitative estimates, I'm afraid the Jason estimates are the ones people will use. If Jason is wrong, can the author state an inkling of why it is wrong? If there is no critique of Jason, it becomes the de-facto truth.

This needs a reference as it is so surprisingly small. The Misner $\mathrm{T} \& \mathrm{~W}$ reference only talks about the polarization change of a wave passing through an object, not about the sensitivity of an HFGW detector.

\section{SPESIF}

September 12, 2009

Gertsenshtein (1962) established theoretically that an electromagnetic (EM) wave in the presence of a magnetic field would generate a gravitational wave (GW) and also hypothesized an "inverse Gertsenshtein effect," in which GWs generate EM photons. Such photons are a second-order effect and according to Eq. (7) of Li, et al. (2009) the number of EM photons are "...proportional to the amplitude squared of the relic HFGWs ..." and that it would be necessary to accumulate such EM photons for at least $1.4 \times 10^{16}$ seconds in order to achieve relic HFGW detection (Li et al., 2009). A different effect was suggested theoretically by Li, Tang and Zhao (1992) in which EM photons having the same frequency and direction as the GWs and suitable phase matching as the GWs, interact directly with GWs in a magnetic field and produce "detection" EM photons that signal the presence of relic HFGWs. In the case of this Li theory the number of EM photons is proportional to the amplitude of the relic HFGWs, $A \approx 10^{-30}$, not the square, so that it would be necessary to accumulate such EM photons for only about 1000 seconds in order to achieve relic HFGW detection ( $\mathrm{Li}$ et al., 2008). Based on the Li theory, as described in more detail in Li and Tang (1997); Li, Tang, Luo and Li, (2000), Li, Tang and Shi (2003), Li and Yang (2004), and Li and Baker (2007), Baker developed a detection device (2001), the Li-Baker HFGW detector (Baker, 2006; Baker, Stephenson and $\mathrm{Li}, 2008$ ). The JASON report (Eardley, 2008) confuses the two effects and erroneously suggests that the Li-Baker HFGW Detector utilizes the inverse Gertsenshtein effect. It does not and does have a sensitivity that is about $A / A^{2}=10^{30}$ greater than that incorrectly assumed in the JASON report.

From: gar@ias-spes.org [mailto:gar@ias-spes.org]

Sent: Monday, September 21, 2009 8:15 AM

To: DrRobertBaker@GravWave.com

Subject: SPESIF-2010: Log\# 001

Dr. Baker,

Your paper Log\# 001: “Applications of High-Frequency Gravitational Waves to the Global War on Terror"

has been approved.

SPESIF 


\title{
Applications of High-Frequency Gravitational Waves to the Global War on Terror
}

\author{
Robert M L Baker, Jr. \\ GravWave ${ }^{\circledR}$ LLC and Transportation Sciences Corporation \\ 8123 Tuscany Ave. \\ Playa del Rey, CA 90293 \\ 310-823-4143;DrRobertBaker@GravWave.com
}

\begin{abstract}
Applications of high-frequency gravitational waves or HFGWs to the global war on terror are now realistic because technology developed by GravWave ${ }^{\circledR}$ LLC and other institutions overseas can lead to devices, some already constructed, that can generate and detect HFGWs. In fact, three HFGW detectors have been built outside the United States and an ultra high-sensitive Li-Baker HFGW Detector has been proposed. HFGW generators have been proposed theoretically by the Russians, Germans, Italians and Chinese. Because of their unique characteristics, such as their ability to pass through all material without attenuation, HFGWs could be utilized for uninterruptible, very low-probability-of-intercept (LPI), high-bandwidth communications among and between anti-terrorist assets. One such communications system, which can be constructed from offthe-shelf elements, is discussed. The HFGW generation device or transmitter alternative selected is based upon bands of piezoelectric crystal, film-bulk acoustic resonators or FBARs energized by conventional Magnetrons. The system is theoretically capable of transmitting and detecting, through use of the Li-Baker HFGW detector, a signal generated on the opposite side of the Earth. Although HFGWs do not interact with and are not absorbed by ordinary matter, their presence can be detected by their distortion of spacetime as measured by the Laser Interferometer Gravitational Observatory (LIGO), Virgo, GEO600, et al., by detection photons generated from electromagnetic beams having the same frequency, direction and phase as the HFGWs in a superimposed magnetic field (Li-Baker HFGW Detector), by the change in polarization HFGWs produce in a microwave guide (Birmingham University Detector) and by other such instruments. Potential theoretical applications, which may or may not be practical yet theoretically possible, are propulsion, including "moving" space objects such as missiles, anti-missiles and warheads in flight; surveillance through buildings and the Earth itself and remote initiation of nuclear events. Such applications can only be quantified and established as practical by the proof-of-concept generation and detection of HFGWs in the laboratory experiment. These important potential HFGW applications are motivations for HFGW research and development and such an R\&D program is recommended for immediate initiation.
\end{abstract}

Keywords: Gravitational Waves, High Frequency Gravitational Waves, National Security, Terrorism, Missile Defense, Foreign Technology

PACS: $95.55 . \mathrm{Ym}, 04.30 . \mathrm{w}, 04.30 . \mathrm{Tv}, 04.80 . \mathrm{Nn}$

\section{INTRODUCTION}

Applications of high-frequency gravitational waves or HFGWs to the global war on terror are now realistic due to technology developed by GravWave ${ }^{\circledR}$ LLC and other institutions overseas and can lead to devices, some already constructed outside the United States, that can generate and detect HFGWs. Because of their unique characteristics, such as their ability to pass through all material without attenuation, HFGWs could be utilized for uninterruptible, very low-probability-of-intercept (LPI), high-bandwidth communications among and between anti-terrorist assets. Although HFGWs do not interact with and are not absorbed by ordinary matter, their presence can be detected by their distortion of spacetime as measured for lowfrequency gravitational waves (LFGWs) by the Laser Interferometer Gravitational Observatory or LIGO (Abbott, et al., 2008), Virgo (Ballardio, et al., 2001), GEO600 (Hogan, 2008) and for HFGWs by detection photons generated from electromagnetic beams having the same frequency, direction and phase as the HFGWs in a superimposed magnetic field, the Li-Baker HFGW Detector (Baker, Stephenson and Li, 2008; Li et al., 2008; Li et al., 2009), by the change in polarization they produce in a microwave-guide loop as in the Birmingham University Detector (Cruise and Ingley, 2005) and by other such HFGW detection instruments (Chincarini and Gemme, 2003; Nishizawa et al., 2008). None of these effects utilized for 
detection represent interaction with matter, but with fields and do not attenuate the GWs. There are a number of alternative devices theorized to generate HFGWs in the laboratory such as the Russians: Grishchuk and Sazhin (1974), Braginsky and Rudenko (1978), Rudenko (2003), Kolosnitsyn and Rudenko (2007); the Germans: Romero and Dehnen (1981) and Dehnen and Romero-Borja (2003); the Italians: Pinto and Rotoli (1988), Fontana (2004); Fontana and Baker (2006); the Chinese: Baker, Li and Li (2006). The HFGW generation device or transmitter alternative selected is based upon bands of piezoelectriccrystal, film-bulk acoustic resonators or FBARs (Baker, Woods and $\mathrm{Li}, 2006$ ) since they are readily available "off the shelf." Additional potential theoretical HFGW applications include propulsion (Section 108 of Landau and Lifshitz, 1975), including "moving" space objects such as missiles, missile warheads, anti-ballistic missiles, etc. in flight (Baker, 2007a), surveillance through buildings and the Earth itself (Baker, 2007b) and remote initiation of nuclear events (Fontana. and Baker, 2007). In order to quantitatively explore these qualitative theoretical studies a proof-of-concept laboratory HFGW generationdetection experiment must be accomplished. These important potential applications to the global war on terror are motivations for HFGW research and development. It should also be recognized that there have been some five decades of theoretical research concerning HFGWs - most of them in the form of hundreds of peer-reviewed publications in the open scientific literature. For a list of these references please visit: http://www.gravwave.com/docs/HFGW\%20References.pdf. In addition three HFGW detectors have been built outside the United States already (Cruise and Ingley, 2005; Chincarini and Gemme, 2003; Nishizawa et al., 2008). Although most of the theoretical applications are stunning, the field of HFGW research is far from being science fiction. The plausibility of the theoretical applications cannot, however, be adequately determined until after a proof-of-concept test is successfully completed.

\section{COMMUNICATIONS}

Of the applications of high-frequency gravitational waves (HFGWs), communication appears to be the most important and most immediate. Gravitational waves have a very low cross section for absorption by normal matter, so HFGWs could, in principle, carry significant information content with effectively no absorption unlike electromagnetic (EM) waves. Multi-channel HFGW communications can be both pointto-point (for example, to deeply submerged submarines) and point-to-multipoint, like cell phones and interconnect the global anti-terrorist assets. For example, one could communicate directly through the Earth from Moscow in Russia to Caracas in Venezuela, as noted in Figure 1, without the need for fiber optic cables, microwave relays, or satellite transponders. Antennas, cables and phone lines would be things of the past. A timing standard alone, provided by HFGW stations around the globe, could result in a multi-billion dollar savings in conventional telecom systems over ten years according to the analysis of Harper and Stephenson (2007). The communication and navigation needs of future magneto hydrodynamic (MHD) aerospace vehicles, such as the MHD aerodyne (www.mhdprospects.com), which is high in electromagnetic interference, similar to plasma interference seen during entry, would be another possible applications area for HFGW communications. This as well as other applications are only possible after sending a "message" from a HFGW generator or transmitter to a Li-Baker Detector (Baker, Stephenson and Li, 2008; Li et al., 2008; Li et al., 2009) or other sensitive HFGW receiver (Stephenson, 2009a; Cruise and Ingley, 2005; Chincarini and Gemme, 2003; Nishizawa et al., 2008). We will first consider the HFGW detector or receiver.

In connection with HFGW detection it should be recognized that unlike the Gertsenshtein effect, a firstorder perturbative photon flux (PPF), proportional to the amplitude of the gravitational wave (GW) $A$ not $A^{2}$, comprising the detection photons or perturbative photon flux (PPF), will be generated in the $x$-direction. Such Gertsenshtein-effect photons are a second-order effect and according to equation (7) of Li, et al. (2009) the number of EM photons are "...proportional to the amplitude squared of the relic HFGWs ..." and that it would be necessary to accumulate such EM photons for at least $1.4 \times 10^{16}$ seconds in order to achieve relic HFGW detection (Li et al., 2009). A different effect was suggested theoretically by Li, Tang and Zhao (1992) in which EM photons having the same frequency and direction as the GWs and suitable phase matching as the GWs, interact directly with GWs in a magnetic field and produce "detection" EM photons that signal the presence of relic HFGWs. The "Li Theory" was validated by eight journal articles; independently peer reviewed by scientists presumably well versed in general relativity, ( $\mathrm{Li}$, Tang and Zhao, 1992; Li and Tang, 1997; Li, Tang, Luo, 2000; Li, Tang and Shi, 2003; Li and Yang, 2004; Li and Baker, 
2007; Li, et al., 2008; Li, et al., 2009). In the case of this Li theory the number of EM photons is proportional to the amplitude of the relic HFGWs, $A \approx 10^{-30}$, not the square, so that it would be necessary to accumulate such EM photons for only about 1000 seconds in order to achieve relic HFGW detection (Li et al., 2008). Based on the Li theory, as described in more detail in the aforementioned eight references, Baker developed a detection device (2001), the Li-Baker

Operational Capability:

\section{Broadband Communications Worldwide through the Earth}

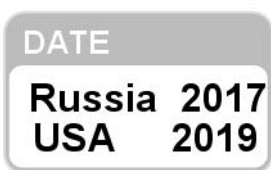

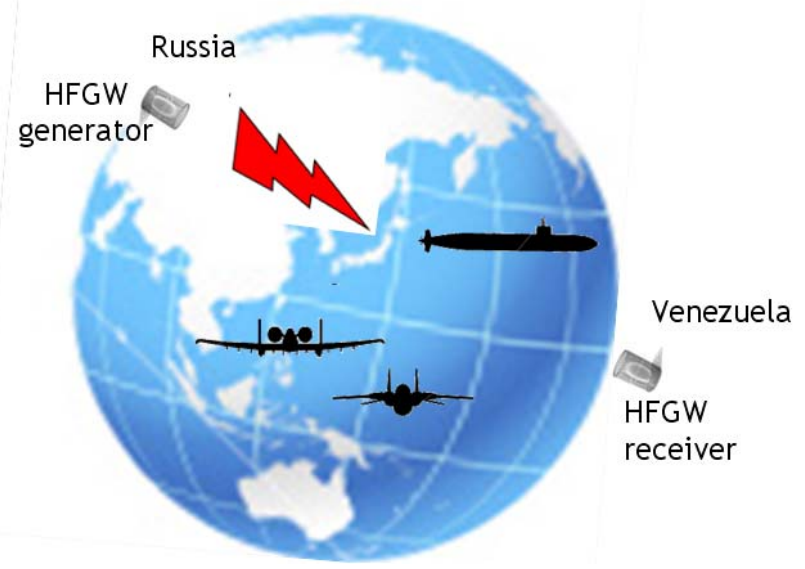

FIGURE 1. HFGW Communication. Operational capability predictions are based on very rough estimates by the author from conversations and impressions gained during three international HFGW Workshops (MITRE2003, Austin 2007 and Huntsville 2009) and trips to China in 2004, 2006 and 2008 and to Europe and the Middle East in 2009.

HFGW detector (Baker, 2006; Baker, Stephenson and Li, 2008). The JASON report (Eardley, 2008) confuses the two effects and erroneously suggests that the Li-Baker HFGW Detector utilizes the inverse Gertsenshtein effect. It does not and does have a theoretical sensitivity that is about $A / A^{2}=10^{30}$ greater than that incorrectly assumed in the JASON report. Noise sources resulting from diffraction from the GB's imperfections and the reflector's edges, blackbody emissions from the enclosure walls (including vibrations and inherent temperature variations), Johnson noise in the HEMIT amplifiers, shot noise in the diffraction fields, noise in the generation of the GB (microwave transmitter noise) and the magnetic field, scattering of the GB (analyzed below), etc. are found to be negligible or suppressed by off-the-shelf microwave absorbers. A major noise-reduction concept for the HFGW detector involves microwave absorbers. Such absorbers are of two types: metamaterial or MM absorbers (Landy, et al., 2008) and the usual commercially available absorbers. In theory multiple layers of metamaterials could result a "perfect" absorber (two layers absorb noise to $-45 \mathrm{db}$ according to p. 3 of Landy, et al., 2008), but in practice that might not be possible so a combination of MMs backed up by the commercially available microwave absorbers would be desirable. As Landy, et al. (2008) state: "In this study, we are interested in achieving (absorption) in a single unit cell in the propagation direction. Thus, our MM structure was optimized to maximize the (absorbance) with the restriction of minimizing the thickness. If this constraint is relaxed, impedance matching is possible, and with multiple layers, a perfect (absorbance) can be achieved." As to the commercially available microwave absorbers, there are several available that offer the required low reflectivity. For example ARC Technologies, Cummings Microwave, the ETS Lindgren Rantec Microwave Absorbers to mention only a few. The ETS Lindgren EHP-5PCL absorbing pyramids seem like a good choice. At normal incidence the typical reflectivity is down $-45 \mathrm{db}$ (guarantied $-40 \mathrm{db}$ ). The power for one $10 \mathrm{GHz}$ photon per second is $6.626 \times 10^{-24} \mathrm{~W}$ and if one can tolerate one thousandth of a photon per second for a series of back and forth reflections off the microwave absorbent walls of the detector as the stray radiation (BPF) ricochet in a zigzag path to the detector, then if the stray radiation were 1000 watts the total required db drop should 
Power $\mathrm{db}=10 \log _{10}($ power out $/$ power in $)=10 \log _{10}\left(6.626 \times 10^{-27} / 1000\right)=-290 \mathrm{db}$

so there should be no problem if there were $290 / 40 \approx 7$ reflections of the noise (BPF) off the pyramids without any other absorption required. Note that Eq. (1) provides the needed absorption of the BPF noise before reaching the detector(s) for a full 1000 watts of stray radiation. A possible better approach would be to remove the restriction of minimizing the MM thickness and incorporate them in the absorption process. Let us consider an absorption "mat" consisting of four MM layers, each layer a quarter wavelength from the next (in order to cancel any possible surface reflection) and provide $\mathrm{a}-45 \mathrm{db}-45 \mathrm{db}-45 \mathrm{db}=-135 \mathrm{db}$ absorption. Behind these MM layers would be a sheet of $10 \mathrm{GHz}$ microwave pyramid absorbers providing a $-40 \mathrm{db}$ absorption before reflection back into the four MM layers (Patent Pending). Thus the total absorption would be $-135 \mathrm{db}-40 \mathrm{db}-135 \mathrm{db}=-310 \mathrm{db}$. The absorption mat would cover the detector containment vessel's walls. Of course, only a small fraction of the GB would comprise stray radiation since, as will be shown, there is negligible scattering in the GB itself.

Ideally the Gaussian beam is a culminated beam having distinct edges. In actuality it is not, but falls off exponentially. In the prototype detector under analysis, which has peak sensitivity at $10 \mathrm{GHz}$, , the energy per detection photon is $h v_{e}=6.626 \times 10^{-34}(\mathrm{Js}) \times 10^{10}\left(\mathrm{~s}^{-1}\right)=6.626 \times 10^{-24}(\mathrm{~J})$, so for a $1,000 \mathrm{~W}$ GB, the total photons per second for the entire beam is $1.51 \times 10^{26}$ photons per second. At the 100 -cm-distant microwave receivers, the theoretical GB intensity is reduced to $\exp \left(-2 \times 100^{2} / 4.3^{2}\right)\left(1.51 \times 10^{26}\right)$, which is essentially zero.

With regard to the background photon flux (BPF) or noise BPF from the scattering in the Gaussian beam, we introduce hydrogen or helium into the detector enclosure prior to evacuating it to reduce the molecular cross-section and, therefore, increase the mean free path. The photon mean free path, $l$, for helium gas molecules at a high-vacuum pressure of $7.5 \times 10^{-7}$ Torr $\left(9.86 \times 10^{-10}\right.$ atmospheres) and temperature of $480 \mathrm{mK}$, is given by (diameter $\mathrm{d}$ of a He molecule is $1 \times 10^{-8} \mathrm{~cm}$ ):

$$
l=1 /(n \sigma)=1 /\left(\left[N_{m} P / / T\right]\left[\pi d^{2} / 4\right]\right)=1 /\left(\left[1.51 \times 10^{13}\right]\left[7.85 \times 10^{-17}\right]\right)=844 \mathrm{~cm},
$$

where $N_{m}=$ number of molecules in a $\mathrm{cm}^{3}$ at standard temperature and pressure (STP) $=2.7 \times 10^{19}, P$ is the pressure in atmospheres and $T$ is temperature in degrees Kelvin or the ratio of the temperature at STP to that in the detector. Since $844 \mathrm{~cm}$ is far longer than the $30 \mathrm{~cm}$ long interaction volume, there will be negligible degradation of the EM-GB interaction due to intervening mass. With regard to scattering, $\lambda_{\mathrm{e}}=3$ $\mathrm{cm}=3 \times 10^{8} \AA$ (wavelength of the GB's EM radiation) is very much greater than the diameter of the He molecule $\left(1 \times 10^{-8} \mathrm{~cm}\right)$, so there would be Ralyeigh scattering (caused by particles much smaller than the wavelength of the EM radiation). The average scattering cross section $\left(\sigma_{\text {ray }}\right)$ per $\mathrm{H}_{2}$ molecule (about the same as per $\mathrm{He}_{2}$ molecule) is given by $\sigma_{\text {ray }}\left(\mathrm{H}_{2}\right)=\left(8.48 \times 10^{-13} / \lambda_{\mathrm{e}}^{4}+1.28 \times 10^{-6} / \lambda_{\mathrm{e}}^{6}+1.61 / \lambda_{\mathrm{e}}^{8}\right) \mathrm{cm}^{2}$ (with $\lambda_{\mathrm{e}}$ in $\AA$ ) $=1.047 \times 10^{-46} \mathrm{~cm}^{2}$. Thus the Rayleigh scattering mean free path is

$$
l_{\text {ray }} \approx 1 /\left(n \sigma_{\text {ray }}\right)=1 /\left(\left[N_{m} P / / T\right]\left[\sigma_{\text {ray }}\left(\mathrm{H}_{2}\right)\right]=1 /\left(\left[1.51 \times 10^{13}\right]\left[1.047 \times 10^{-46}\right]\right)=6 \times 10^{32} \mathrm{~cm} .\right.
$$

Utilizing the exponential change in scattering along the Gaussian beam

$$
I=I_{0} e^{-z / r a y},
$$

where $I$ is the intensity of the scattering in photons per second at a distance $\mathrm{z}$ from the GB transmitter and $I_{0}$ is the initial intensity of the GB $=1.51 \times 10^{26} \mathrm{~s}^{-1}$. The interaction volume, where the EM, HFGWs and the magnetic field interact to produce the PPF, extends from $\mathrm{z}=10 \mathrm{~cm}$ to $\mathrm{z}=40 \mathrm{~cm}$, so that the intensity difference between these two points (the scattering from the interaction volume) is $I(10)-I(40)=I_{0}\left(\mathrm{e}^{-10 / \text { ray }}\right.$ $\left.-\mathrm{e}^{-40 / r a y}\right) \approx\left(1.51 \times 10^{26}\right)\left(-1+10 / 6 \times 10^{32}+1-40 / 6 \times 10^{32}\right)=3 \times 10^{-7}$ photons per second scattered in the $30 \mathrm{~cm}$ long interaction volume, which is negligible.

The generation of HFGWs in the laboratory or the HFGW transmitter is based upon the well-known astrodynamic gravitational-wave generation process (Landau and Lifshitz (1975)). In Figure 2 is shown the gravitational wave $(\mathrm{GW})$ radiation pattern for orbiting masses in a single orbit plane where $\mathrm{f}_{c f}$ is the centrifugal force and $\Delta \mathrm{f}_{c f}$ is the change in centrifugal force, acting in opposite directions, at masses $\mathbf{A}$ and B. Next consider a number $N$ of such orbit planes stacked one on top of another again with the gravitational-wave $(\mathrm{GW})$ radiation flux $\left(\mathrm{Wm}^{-2}\right)$ growing as the $\mathrm{GW}$ moves up the axis of the $N$ orbit planes as in Figure 3 . We now replace the stack of orbital planes by a stack of $N$ HFGW-generation elements. 
These elements could be pairs of laser targets (Baker, Li and Li, 2006), gas molecules (Woods and Baker, 2009), piezoelectric crystal pairs (Romero-Borja and Dehnen, 1981; Dehnen and Romero-Borja, 2003) or film-bulk acoustic resonator (FBAR) pairs, which also are composed of piezoelectric crystals (Woods and Baker, 2005). Since they can be obtained off the shelf we select the FBAR alternative. Thus we now have a HFGW wave moving up the centerline of the FBAR-pair tracks, as shown in Figure 1 of Baker (2009).

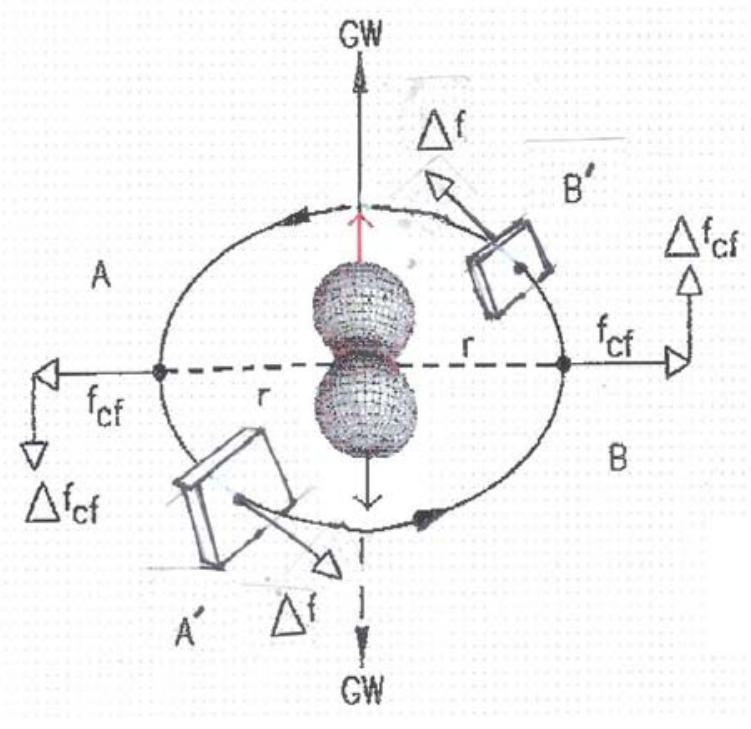

FIGURE 2. Radiation pattern calculated by Section 110 Page 356.

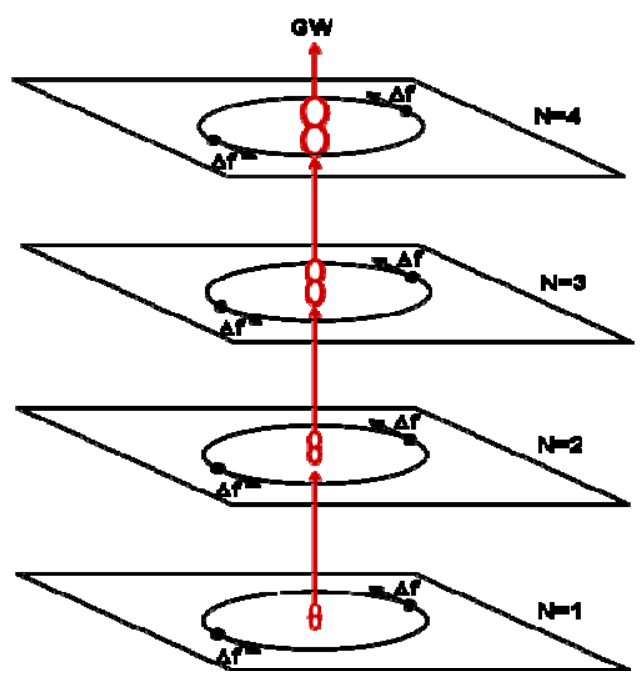

FIGURE 3. GW Flux Growth Analogous to Stack of $N$ Orbital Planes

The HFGW flux or signal increases in proportion to the square of the number HFGW-generation elements, $N$ (Scully and Svidzinsky, (2009), The Super of Superradiance," Science 325, pp.1510-151. The $N^{2}$ build up is attributed to two effects: one $N$ from there being $N$ HFGW power sources or generation elements and the other $N$ from the narrowing of the beam so that the HFGW is more concentrated and the flux $\left(\mathrm{Wm}^{-2}\right)$ thereby increased (Romero-Borja and Dehnen, 1981; Dehnen and Romero-Borja, 2003). Note that it is not necessary to have the FBAR tracks perfectly aligned (that is the FBARs exactly across from each other) since it is only necessary that the energizing wave front (from Magnetrons in the case of the FBARs as in Baker, Woods and Li (2006)) reaches a couple of nearly opposite FBARs at the same time. The HFGW beam is very narrow, usually less than $10^{-4}$ radians (Baker and Black, 2009) and increasing $N$ narrows the 
beam. Additionally multiple HFGW carrier frequencies can be used, so the signal is very difficult to intercept by terrorists, and is therefore useful as a low-probability-of-intercept (LPI) signal, even with widespread adoption of the technology.

The force change, $\Delta \mathrm{f}$, produced by a single off-the-shelf FBAR is $2 \mathrm{~N}$ (for $1.8 \times 10^{8}$ FBARS the force change is $4 \times 10^{8} \mathrm{~N}$ or about $2 \mathrm{~N}$ per FBAR according to Woods and Baker (2005)). The basic equation for the GW power produced by a change-in-force pair such as FBARs, $P$, as derived in Baker (2006), is:

$$
P=1.76 \times 10^{-52}(2 r \Delta \mathrm{f} / \Delta t)^{2} \quad \mathrm{~W},
$$

where $2 r$ is the distance between the FBAR pair, $\mathrm{m}, \Delta \mathrm{f}$ is the force change, $\mathrm{N}$ and $\Delta t$ is the time over which the force change occurs, $s$ or the inverse of the HFGW frequency, $1 / v_{\mathrm{GW}}$. As can be seen from Figure 2 the fixed (not orbiting) FBARs are faced (i.e., the normal to their flat surface in the $\Delta$ f direction) tangent to the circle at A' and B'. From p. 1282 of Baker, Woods and Li (2006) in plan form the flat surface is $100 \mu \mathrm{m} \times 100 \mu \mathrm{m}$ and they are about $1 \mu \mathrm{m}$ thick. To allow for margins we will take the FBAR dimensions overall as $110 \times 110 \times 2 \mu \mathrm{m}^{3}$. Let $n$ FBARs be spread out radially like a vane in a double-helix configuration (Figure 3 of Baker and Black, 2009). Thus $\Delta \mathrm{f}=2 \mathrm{n} \mathrm{N}$. If $n=1000$, then the radial extent of the FBARs vane would be $11 \mathrm{~cm}$. For $r=1 \mathrm{~m}, \Delta \mathrm{f}=2000 \mathrm{~N}$ and $v_{\mathrm{GW}}=4.9 \mathrm{GHz}$, the HFGW generated by the $\mathrm{i}^{\text {th }} \mathrm{FBAR}$ vane pair is $P_{i}=6.76 \times 10^{-26} \mathrm{~W}$. Note that $2 r=2 \mathrm{~m}$ is greater than the HFGW wavelength $\lambda_{\mathrm{GW}}$ $=6.1 \mathrm{~cm}$. Nevertheless, according to page 1283 of Baker, Woods and Li (2006) equation (1) is still approximately valid. From equation (6) and Table 2 (for $10^{\circ}$ half angle at $N=1$ ) of Baker and Black (2009) we have for the signal, $S(1.0)$, or flux, $\mathrm{F}(1.0)$, at one meter from the end of an array of $N$ FBAR vane pairs

$$
S(1.0)=\mathrm{F}(1.0)=N^{2} \mathrm{~F}(1.0)_{\mathrm{N}=1}=N^{2}(0.336) P_{\mathrm{i}}
$$

Let us place the FBAR vane pairs adjacent to each other so that there will be $2 \pi \mathrm{r} / 2 \mu=3.14 \times 10^{6}$ vane pairs on each $110 \mu \mathrm{m}$ thick level leading up a cylindrical FBAR array (US Patents 6,417,597 and 6,784,591 and Patents Pending). We will "stack" these $110 \mu$ thick levels one on top of the other in a double helix configuration (Baker and Black, 2009) in order to increase $N$ and narrow the beam. There will be $10 \mathrm{~m} / 110$ $\mu \mathrm{m}=9.1 \times 10^{4}$ levels so that $N=2.9 \times 10^{11}$. Thus, from equations (5) and (6), we have $S=1.9 \times 10^{-3} \mathrm{Wm}^{-2}$ at a one meter distance or if we were $1.3 \times 10^{7} \mathrm{~m}$ (diameter of Earth) distance, then $S=1.12 \times 10^{-17} \mathrm{Wm}^{-2}$. From equation (7) below, derived in the Appendix of Baker, Stephenson and Li (2008), the amplitude $A$ of the $\mathrm{HFGW}$ is given by:

$$
A=1.28 \times 10^{-18} \sqrt{\mathrm{S}} / v_{\mathrm{GW}} \mathrm{m} / \mathrm{m},
$$

so that $A=0.88 \times 10^{-36} \mathrm{~m} / \mathrm{m}$. The sensitivity of the Li-Baker HFGW detector is on the order of $10^{-32} \mathrm{~m} / \mathrm{m}$, but its sensitivity can be increased dramatically (Li and Baker, 2007) by introducing superconductor resonance chambers into the interaction volume (which also improves the Standard Quantum Limit) and two others between the interaction volume and the two microwave receivers. Together they provide an increase in sensitivity of five orders of magnitude and result in a sensitivity of the Li-Baker detector to HFGWs having amplitudes of $10^{-37} \mathrm{~m} / \mathrm{m}$. Since the exact frequency and phase of the HFGW signal is known (unlike bigbang relic HFGWs, for which the Li-Baker detector was designed as shown in Figure 4 from Grishchuk (2008) that exhibits the $10 \mathrm{GHz}$ peak in relic HFGW energy density), a much more sensitive, optimized HFGW detector will likely be developed. Such a sensitive detector will still not be quantum limited (Stephenson, 2009b). The power required at $2 \times 56 \mathrm{~mW}$ per FBAR pair (Woods and Baker, 2005) would be about $2 \times n \times N \times 56 \times 10^{-3}=3.2 \times 10^{13} \mathrm{~W}$. Thus to reduce the average power to, say $32 \mathrm{MW}$ one could communicate with one microsecond bursts every second (roughly a $49 \mathrm{kHz}$ information bandwidth). One would still need about 32 thousand off-the-shelf Microwave-Oven-type, in-phase, one kW Magnetrons distributed along the double-helix cylinder walls. The Magnetron would be angled up the double helix along the direction of the HFGW beam and produce about a kilowatt of average power but with MW burst capability. The frequency-standard optimized FBARs would be replaced by $\Delta f$ optimized ones. The cost should be less than 20 to 30 million dollars US or a small fraction of the cost of LIGO, Virgo and GEO600 detectors. The very speculative use of superconductor GW lenses (US Patent 6,784,591) and mirrors (such mirrors suggested by Baker (2003), Woods (2006), Chiao, et al., (2009) and Minter, et al. (2009), but in a concave parabolic form (Baker, 2003 and 2005)) would serve to further concentrate the HFGWs and increase their amplitude $A$ at the detector/receiver and greatly improve the information bandwidth. 


\section{SURVIELANCE}

The potential for through-earth or through-water observations by utilizing the extreme sensitivity of HFGW generation-detection systems to polarization angle changes (possibly sensitive to even less than $10^{-4}$ to $10^{-40}$ radians change) might allow for observing subterranean structures and geological formations (such as oil deposits); creating a transparent ocean; viewing three-dimensional building interiors; viewing terrorist buried devices, hidden missiles and weapons of mass destruction; achieving remote acoustical surveillance or eavesdropping, etc., or even a full-body scan without radiation danger (Baker 2007a). Please see Figure 4. Note that it is not necessary to measure the polarization, only to sense a difference. Long-wavelength gravitational waves have thousand- to million-meter wavelengths, but these are of no practical surveillance value, due to their diffraction and resulting poor resolution. It should also be noted that HFGW imaging could, in theory, defeat the recently proposed EM cloaking or stealth techniques (Leohart (2006); Pendry, Schung and Smith (2006)) if these techniques are ever practically applied. It will not be possible to prove or absolutely disprove the potential for this very theoretical HFGW terrorist surveillance application until after the proof-of-concept experimental results of the HFGW laboratory generation-detection experiment are analyzed, with various material placed between the HFGW generator and detector. As previously noted gravitational waves, including HFGWs, pass through most material with little or no attenuation; but although they are not absorbed, their polarization, phase, velocity (causing refraction or bending of gravitational rays), backscatter, and/or other characteristics can be modified by a material object's texture and internal structure. For example, the change in polarization of a GW passing through a material object is discussed in Misner, Thorne and Wheeler (1973): "In the real universe there are spacetime curvatures due not only to the energy of gravitational waves, but also more importantly to the material [objects and structures] content of the universe ... its wavelength changes [based on gravitational red shift] and [the gravitational wave] backscatters off the curvature to some extent. If the wave is a pulse, then the backscatter will (change) its shape and polarization...." It is extremely difficult to theoretically establish the actual magnitude of the changes, especially at very high frequencies $\left(10^{9} \mathrm{~Hz}\right.$ and higher) and to quantify them prior to the proof-of-concept HFGW generation/detection laboratory experiments.

\section{REMOTE DISPLACEMENT OF MASSES}

HFGWs could theoretically be used for remote displacement of masses and control of the motion of objects such as missiles, missile warheads (please see Figure 5), anti-missiles, spacecraft, and asteroids, and remote control of clouds of hazardous vapors deployed by terrorists. From section 108, page 349 of Landau and Lifshitz (1975):

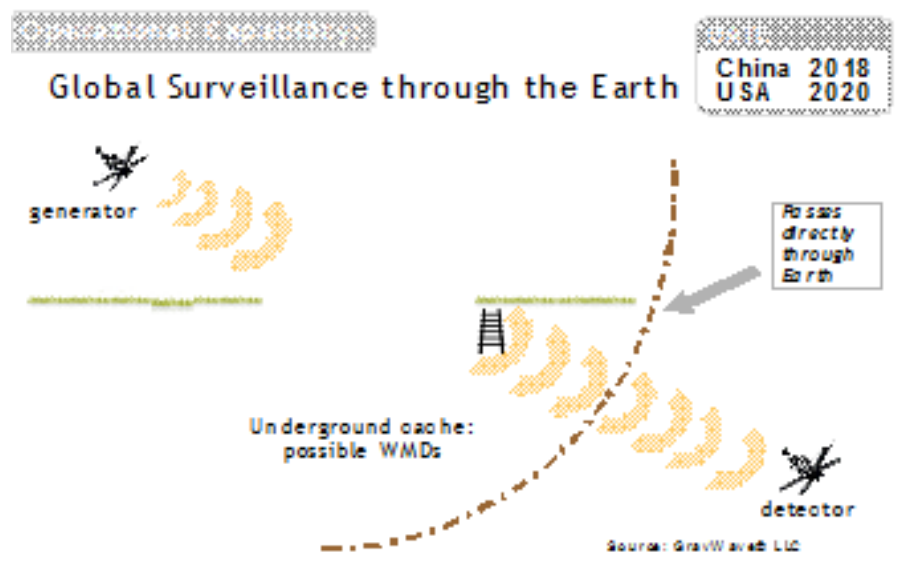

FIGURE 4. HFGW Surveillance. Operational capability predictions are based on very rough estimates by the author from conversations and impressions gained during three international HFGW Workshops (MITRE2003, Austin 2007 and Huntsville 2009) and trips to China in 2004, 2006 and 2008 and to Europe and the Middle East in 2009. 
"Since it has definite energy, the gravitational wave is itself is the source of some additional gravitational field [static g-field]. Like the energy producing it, this field is a second-order effect in the $h_{i k}$. But in the case of high-frequency gravitational waves the effect is significantly strengthened: the fact that the pseudotensor $t^{i k}$ is quadratic in the derivatives of the $h_{i k}$ introduces the large factor $\lambda^{-2}$. In such a case we may say that the wave itself produces the background field [static g-field] on which it propagates. This [static g] field is conveniently treated by carrying out the averaging described above over regions of fourspace with dimensions large compared to $\lambda$. Such an averaging, smoothes out the short-wave 'ripple' and leaves the slowly varying background metric [static g-field]." (Brackets and italics added for clarity and emphasis.) This is a qualitative theory; but does indicate a dependence of the effect on the square of the frequency so it should be significant for HFGWs. A quantitative analysis must necessarily await a laboratory HFGW generation-detection experiment in order to determine the value of parameters involved. Nevertheless, one can hypothesize the effect assuming certain perturbations as follows:

Gravitational field changes by one or more HFGW generators could urge a spacecraft in a given direction, causing a lower static gravitational field in front of a vehicle (it "falls" forward) and a higher one behind (providing a "push"). The concept is that the mass essentially "rolls" down a "hill" produced by the static g-field; that is, potential energy increase of a mass is provided by the energetic HFGWs. As was noted, the magnitude of the static g-field is proportional to the square of the HFGW frequency (Landau and Lifshitz, 1975 ) and the effect is described in U. S. Patent Application, Baker (2007b). Tests with $10^{9} \mathrm{~Hz}$ or higher gravitational waves must be accomplished before the application is either discarded or accepted.
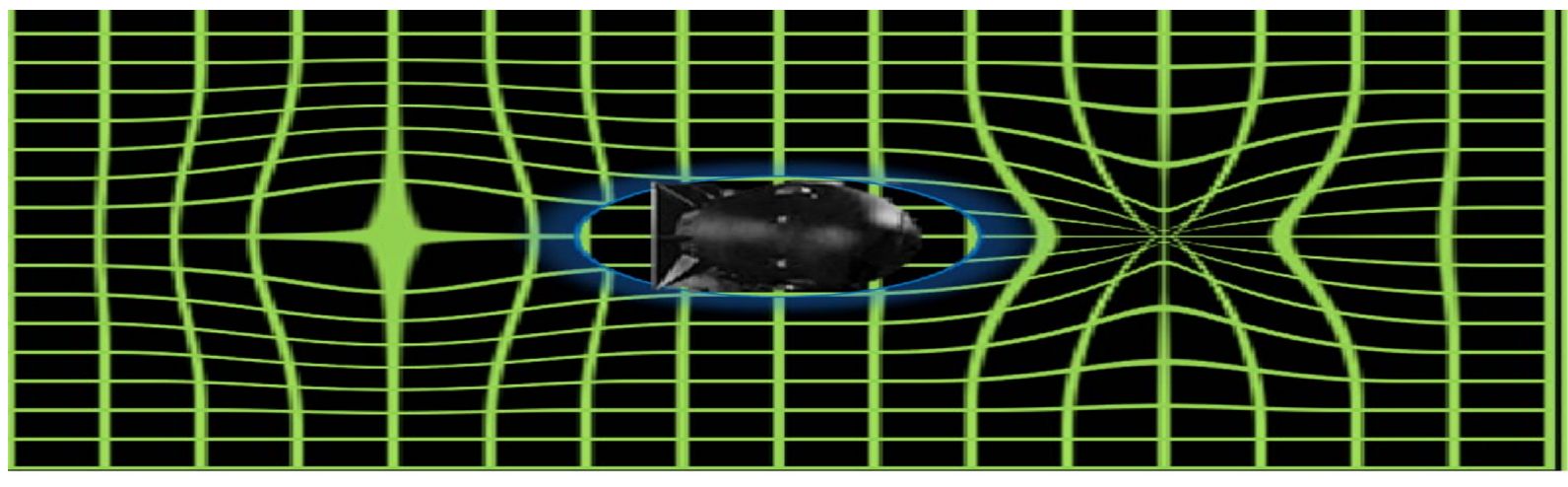

FiGURE 5 Missile warhead moved or displaced by HFGWs (Landau and Lifshitz (1975)).

As suggested by Fontana (2004): “A large literature exists on colliding gravitational waves (Szekeres, 1972). It has been found that the collision or focusing (Alekseev and Griffiths, 1995 and 1996) of gravitational waves produce curvature singularities. These singularities have properties very similar to those of a black hole, an essential and fundamentally simple object, which produces a gravitational field. Gravitational wave propulsion is the application of these theories to space travel. Generators of GWs could be installed directly onboard or beamed remotely to a spacecraft to induce curvature singularities near the spacecraft. The use of HFGW '.. as a source of some additional gravitational field...' at a distance was suggested by Landau and Lifshitz (1975). According to General Relativity, spacecraft mass interacts with spacetime curvature, therefore the spacecraft will move towards the singularity. In the Newtonian picture, because of the non-linearity of space, the wave at the focus is converted to a Coulomb-like gravitational field." Until an experiment provides actual data, we only know theoretically that the static g-field increases with the square of the HFGW frequency. Its persistence may be related to the amplitude of the HFGW and its extent is dependent on the size of the intersecting HFGW beams. We would utilize HFGW frequencies equal to or higher than those utilized for HFGW communications, $v_{\mathrm{GW}}=4.9 \times 10^{9} \mathrm{~s}^{-1}$. According to p. 175 of Baker and Makemson (1960) a perturbative derivative of the vis-viva equation from celestial mechanics yields

$$
2 \mathrm{~s}^{\cdot} \cdot{ }^{\prime}=\mu \mathrm{a}^{\prime} / \mathrm{a}^{2}
$$


where $\mathrm{s}^{\prime}$ is the missile's speed, $\mathrm{s}^{.}$is the perturbation in speed (i.e., perturbative acceleration caused by the static g-field change), $\mu=1$ in characteristic units and a' is the perturbation in the trajectory's semi-major axis a. Thus the perturbative change in a due to the g-field change is

$$
a^{\prime}=2 s^{\prime} \cdot a^{2} \text {. }
$$

Using the standard astrodynamics equations found, for example, on pages 90 and 91 of Herrick (1971), a computer program (to be found below), yields from a 26.8 to a 2.7 mile perturbative g-field change in missile entry location for 6,200 mile ICBM trajectories (with 50 to 100 mile length, 0.1 to $0.01 \mathrm{~g}$-field perturbations). For short-range 1,400 mile trajectories, it yields from a 2.0 to a 0.41 mile perturbative gfield change in missile entry location (with 25 to 50 mile length, 0.1 to 0.01 g-field perturbations). Such modest changes would not greatly reduce the damage caused by terrorist ICBM nuclear strikes, but would frustrate anti-missile systems (by perturbing their trajectories) or defend against, for example, surgical strikes against submerged submarine assets. The computer program, which is meant to be a tool for orderof-magnitude calculation (the parameters of which would come from HFGW experiments) in True BASIC follows:

Print "This program computes the change in Missile entry location caused by a "

Print " HFGW-produced g-field change for minimum-velocity missile trajectories."

REM Refer to pp. 91 and 92 of Herrick (1971)

Print "What is the geocentric angle between launch and entry in degrees?"

Input delta_v

Let range $=2 *$ PI*3963* delta_v/360

Print "Range in miles $=$ ",range

Print "What is impact latitude in degrees?"

Input impact latitude

Print "What is the length of the trajectory segment of the g-field change in miles?"

Input g_field_length ! miles

Print "What is the magnitude of the g-field change at launch in g's ?"

Input g_field

Let s_dot_grav $=\mathrm{g}$ _field

! g's

OPTION ANGLE degrees

Let earth_speed_at_impact $=17.4 *$ COS(impact_latitude)

Let gamma sub zero $=45$ - delta_v/4

Let $\mathrm{e}=\mathrm{TAN}($ gamma_sub_zero)

Let $\mathrm{a}=1 /\left(1+\mathrm{e}^{\wedge} 2\right)$

Let $\mathrm{sdot}=\operatorname{SQR}\left(1-\mathrm{e}^{\wedge} 2\right)$

Let initial_speed $=$ sdot*4.912

! degrees

Let $\mathrm{RA}=\mathrm{a}^{*}(1+\mathrm{e})$

Print "Height in miles at apogee ",3963*(RA - 1)

OPTION ANGLE radians

Let cos_E_0 = -e

Let sine E_ $0=\operatorname{SQR}\left(1-\mathrm{e}^{\wedge} 2\right)$

Let $\mathrm{E} 0=\overline{\mathrm{A} C O S}(\cos \mathrm{E} 0)$

Let M_0 = E_0 - e*sine_E_0

Let $\mathrm{n}=0.07 \overline{33} 67 /\left(\mathrm{a}^{\wedge} 1.5\right)$

Let flight_time $=\left(2 * \mathrm{PI}-2 * \mathrm{M} \_0\right) / \mathrm{n}$

! perturbative accel.

Print "The trajectory flight time in minutes from launch to entry/impact $=$ ",

Let perturbative derivative $\mathrm{a}=2 * \mathrm{a}^{\wedge} 2 * \operatorname{sdot}^{*} \mathrm{~s}$ dot grav ! characteristic units

Let pertubatve_time_interval $=$ g_field_length $/$ initial_speed ! seconds

Print "The time the perturbation at launch acts in seconds $=$ ", pertubatve_time_interval

Let pertubatve time interval $=$ pertubatve time interval $/(13.447 * 60) !$ secs per radian

Let delta_a $=$ perturbative_derivative_a $*$ pertubatve_time_interval

Print "delta a change due to launch g-field perturbation $="$, delta_a $\quad$ ! earth radii

Let fractional_orbit_scale_change $=$ delta_a/a

Let range_change $=$ range* fractional_orbit_scale_change

Print "Perturbative g-field scale change in Missile impact in miles =", range_change

Let Period $=84.49^{*} \mathrm{a}^{\wedge} 1.5 \quad$ ! minutes

Let fraction_orbit_period_change $=$ Period $^{*}$ fractional_orbit_scale_change

Let fraction_flight_time $=$ flight time/Period ! minutes

Let fraction_flight_time_change $=$ fraction_flight_time* fraction_orbit_period_change 
Let impact location change $=$ earth speed at impact* fraction flight time-change Print "Impact location change in miles due to Earth rotation $="$, impact_location_change end

With regard to more conventional HFGW propulsion, a very well known example of the rocket propulsion effect that can be produced by gravitational waves is that of a star undergoing asymmetric octupole collapse, which achieves a net velocity change of 100 to $300 \mathrm{~km} / \mathrm{s}$ via the anisotropic emission of gravitational waves (Bekenstein, 1973). Bonnor and Piper (1978) performed a rigorous analysis for their study of gravitational wave rockets. They obtained the gravitational wave rocket equations of motion directly by solving the Einstein general relativistic field equation in a vacuum using the spacetime metric of a photon rocket as a model. The photon fluid stress-energy tensor for the photon rocket model must be cancelled out so that one actually solves the Einstein vacuum field equation because the gravitational waves that propel the rocket are not a physical fluid. Instead, they are ripples in the shape of spacetime that move through the surrounding background spacetime. So Bonner and Piper added new terms within the resulting vacuum field equation that cancel out the photon fluid stress-energy tensor in order to arrive at the equations of motion. To carry out their program, they found that a gravitational source looses mass by the emission of quadrupole waves and gains momentum from recoil, when it emits quadrupole and octupole waves. Thus, the terms that they added to the photon rocket metric are those representing quadrupole and octupole gravitational waves. A gravitational wave rocket will perform exactly like a photon rocket (Davis, 2009). It will have the maximum possible specific impulse with light-speed exhaust velocity because gravitational waves propagate through space at the speed of light. But such rockets also have extremely low thrust (similar to EM propulsion), and so would be more applicable for interstellar missions rather than interplanetary missions within our solar system.

\section{CONCLUSIONS}

High-frequency gravitational wave (HFGW) generators have been proposed theoretically by the Russians, Germans, Italians and Chinese. HFGW detectors are a reality and three have been actually constructed outside the United States by the English, Italians and Japanese. A theoretically more sensitive detector than these, the Li-Baker, utilizing metamaterial and off-the-shelf microwave absorbers to eliminate noise, together with a theoretical, multi-FBAR HFGW generator in a double-helix configuration that are discussed, could be utilized for transglobal, low-probability of intercept (PPI) communications among antiterrorist assets. The multi-elements of the transmitter (HFGW generator) are off-the-shelf piezoelectric film-bulk acoustic resonators or FBARs energized by off-the-shelf' modified Magnetrons. In theory a large number of these FBAR elements could lead to HFGW generator-detector communications for a laboratory proof-of-concept experiment. Pending the recommended proof-of-concept HFGW experiment other HFGW applications could be of value to the global war on terror. These theoretical applications, yet to be quantified, but discussed herein, include surveillance and remote displacement of masses such as missiles and anti-missiles.

\section{ACKNOWLEDGMENT}

The assistance in the preparation of the figures by Amara D. Angelica and Christine S. Black is gratefully acknowledged. Transportation Sciences Corporation supported travel utilized to obtain operational capability predictions for many of the HFGW applications-implementations outside of the United States. 


\section{REFERENCES}

Abbott, B. et al., "Searching for a Stochastic Background of Gravitational Waves with the Laser Interferometer Gravitational Wave Observatory," The Astrophysical Journal 659, (2007), pp. 918-930.

Alekseev, G. A. and Griffiths, J. B., "Gravitational waves with spherical wavefronts," Class. and Quant. Grav. 12, (1995), pp. L13-L18.

Alekseev, G. A. and Griffiths J. B., "Exact solutions for gravitational waves with cylindrical, spherical and toroidal wavefronts," Class. and Quant. Grav. 13, (1996), pp. 2191-2209.

Baker R. M L, Jr. and Makemson, M. W., An Introduction to Astrodynamics, Academic Press, New York and London, (1960).

Baker, R. M L, Jr., Peoples Republic of China Patent Number 01814223.0, “Gravitational Wave Generator,” filed July 13, 2001 and granted September 2, 2005, (2001).

Baker, R. M L, Jr., "Application of High-Frequency Gravitational Waves to Imaging," paper HFGW-03-120, Gravitational-Wave Conference, The MITRE Corporation, May 6-9, (2003), pp. 2-4.

Baker, R. M L, Jr., "Applications of High-Frequency Gravitational Waves (HFGWs)," in the Proceedings of the Space Technology and Applications International Forum (STAIF-2005), edited by M.S. El-Genk, American Institute of Physics Conference Proceedings 746, Melville, New York, (2005), pp. 1308-1309.

Baker, R. M L, Jr., "Novel formulation of the quadrupole equation for potential stellar gravitational-wave power estimation," Astronomische Nachrichten 327, No. 7, (2006), pp. 710-713.

Baker, R. M L, Jr.., Li, F. Y. and Li, R., "Ultra-High-Intensity Lasers for Gravitational Wave Generation and Detection" in the Proceedings of the Space Technology and Applications International Forum (STAIF-2006), edited by M.S. El-Genk, American Institute of Physics Conference Proceedings 813, Melville, New York, (2006), pp.1249-1258. Available at:

http://www.drrobertbaker.com/docs/AIP;\%20HFGW\%20Laser\%20Generator.pdf

Baker, R. M L, Jr., Woods, R. C. and Li, F. Y., "Piezoelectric-Crystal-Resonator High-Frequency Gravitational Wave Generation and Synchro-Resonance Detection," in the Proceedings of the Space Technology and Applications International Forum (STAIF-2006), edited by M.S. El-Genk, American Institute of Physics Conference Proceedings 813, Melville New York, (2006), pp. 1280-1289. Available at: http://www.drrobertbaker.com/docs/AIP;\%20HFGW\%20Piezoelectric\%20Generator.pdf

Baker, R. M L, Jr., United States Patent Application Number 11/173,080, “Gravitational Wave Propulsion,” publication date, January 4. (2007a).

Baker, R. M L, Jr., "Surveillance Applications of High-Frequency Gravitational Waves," in the Proceedings of the Space Technology and Applications International Forum (STAIF-2007), edited by M.S. ElGenk, American Institute of Physics Conference Proceedings 880, Melville, New York, (2007b), pp.10171026. Available at: http://www.gravwave.com/docs/AIP;\%20HFGW\%20Surviellance.pdf

Baker, R. M L, Jr., Stephenson, G.V. and Li, F.Y., "Proposed ultra-high sensitivity HFGW Detector," in the Proceedings of the Space Technology and Applications Int. Forum (STAIF-2008), edited by M.S. ElGenk, American Institute of Physics Conference Proceedings 969, Melville, New York, (2008), pp. 10451054. Aavailable at: http://www.gravwave.com/docs/Proposed\%20Ultra 08.pdf

Baker R. M L, Jr. and Black, C. S., "Radiation Pattern for a Multiple-Element HFGW Generator," in the Proceedings of the Space, Propulsion and Energy Sciences International Forum (SPESIF), edited by Glen Robertson, American Institute of Physics Conference Proceedings 1103, Melville, New York, (2009), pp. 582-590. Available at: http://www.drrobertbaker.com/docs/Analyses $\% 20$ of $\% 20$ HFGW $\% 20$ Generators $\% 20$ and $\% 20$ Radiation $\% 20 \mathrm{~Pa}$ ttern.pdf

Baker, R. M L, Jr., "Communication Study,” (2009). Available at: http://www.gravwave.com/docs/com\%20study\%20composite.pdf

Baker, R. M L, Jr., "Input Power Requirements for High-Frequency Gravitational Wave Generators," in the Proceedings of the Space, Propulsion and Energy Sciences International Forum (SPESIF), edited by Glen Robertson, American Institute of Physics Conference Proceedings 1103, Melville, New York, (2009), pp. 591-598. Available at: http://www.drrobertbaker.com/docs/HFGW's\%20Power\%20Requirements.pdf

Ballardin, G., "Measurement of the VIRGO superattenuator performance for seismic noise suppression," Rev. Sci. Instrum. 72, (2001), p. 9.

Bekenstein, J. D., “Gravitational-Radiation Recoil and Runaway Black Holes,” Astrophys. J. 183, (1973), pp. 657-664.

Braginsky, V. B. and Rudenko, V. H., "Gravitational waves and the detection of gravitational radiation," Section 7: "Generation of gravitational waves in the laboratory," Physics Report (Review section of Physics Letters), 46, Number 5, (1978), pp. 165-200.

Bonnor, W. B., and Piper, M. S., "The gravitational wave rocket," Class. Quant. Grav. 14, (1978),pp. $2895-2904$.

Chiao, R.Y., Wegter-McNelly , K. and Minter , S. J., "Do Mirrors for Gravitational Waves Exist?" arXiv:0903.0661v1 [gr-qc], March 4, (2009).

Chincarini, A. and Gemme, G., "Micro-wave based High-Frequency Gravitational Wav detector," paper HFGW-03103, Gravitational-Wave Conference, The MITRE Corporation, May 6-9, (2003). 
Cruise, A. M. and Richard M. J. Ingley, R. M. J., "A correlation detector for very high frequency gravitational waves," Class. Quant. Grav. 22, (2005),pp. 5479-5481.

Davis, E. W., "Chapter 4: Gravity Control within Newtonian and General Relativity Physics," Frontiers of Propulsion Science, editors Marc. G. Millis and Eric W. Davis, Progress in Astronautics \& Aeronautics Series 227, American Institute of Aeronautics \& Astronautics Press, Reston, VA, (2009), pp. 175-227.

Dehnen, H. and Romero-Borja, F., "Generation of GHz - THz High-Frequency Gravitational Waves in the laboratory," paper HFGW-03-102, Gravitational-Wave Conference, The MITRE Corporation, (2003). http://www.gravwave.com/docs/Analysis\%20of\%20Lab\%20HFGWs.pdf

Eardley, et al. , "High Frequency Gravitational Waves," JSR-08-506, October, the JASON defense science advisory panel and prepared for the Office of the Director of National Intelligence, (2008).

Fontana, G. , "Design of a Quantum Source of High-Frequency Gravitational Waves (HFGW) and Test Methodology," in the Proceedings of the Space Technology and Applications International Forum (STAIF-2004), edited by M. S. El-Genk, American Institute of Physics 699, Melville, New York, (2004), pp. 1114-1121.

Fontana, G. and Baker, R. M L, Jr., "Generation of Gravitational Waves with Nuclear Reactions," in the proceedings of Space Technology and Applications International Forum (STAIF-2006), edited by M.S. El-Genk, American Institute of Physics Conference Proceedings, Melville NY 813, (2006), pp. 1352-1358. http://www.drrobertbaker.com/docs/AIP;\%20HFGW\%20Nuclear\%20Generator.pdf

Fontana, G. and Baker, R. M L, Jr. , "HFGW-Induced Nuclear Fusion,” in the Proceedings of the Space Technology and Applications International Forum (STAIF-2007), edited by M.S. El-Genk, American Institute of Physics Conference Proceedings 880, Melville, New York, (2007), pp. 1156-1164. http://www.gravwave.com/docs/AIP;\%20HFGW\%20Nuclear\%20Fusion.pdf

Gertsenshtein, M. E, "Wave resonance of light and gravitational waves," Soviet Physics JETP 14 1, (1962), pp. 84-85.

Grishchuk, L. P. and Sazhin, M. V, "Emission of Gravitational Waves by an Electromagnetic Cavity," Sov. Phy.JETP 38, No. 2, (1974), pp. 215-221.

Grishchuk, L.P., "Discovering Relic Gravitational Waves in Cosmic Microwave Background Radiation," Proceedings of the School, Eds. I. Ciufolini and R. Matzner, (in press) Springer (2008), arXiv:0707.3319v3

Harper, C. and Stephenson, G. V. (2007), "The Value Estimation of an HFGW Frequency Time Standard for Telecommunications Network Optimization," in the Proceedings of the Space Technology and Applications International Forum (STAIF-2007), edited by M.S. El-Genk, American Institute of Physics Conference Proceedings 880, Melville, New York, (2007), pp. 1083-1091. http://www.gravwave.com/docs/AIP;\%20HFGW\%20Telecommunications.pdf and http://www.gravwave.com/ppt/HFGW\%20Telecom_files/frame.htm

Hogan, C. J., "Measurement of quantum fluctuations in geometry," Phys, Rev. D 77, (2008), p.104031.

Herrick, S., Astrodynamics, Volume 1, Van Nostrand Reinhold Company, London, (1971).

Kolosnitsyn, N. I. and Rudenko, V. N., "Generation and Detection of the High Frequency Gravitational Radiation in a Strong Magnetic Field," in the proceedings of the HFGW2 Workshop, Institute of Advanced Studies at Austin (IASA), Texas, September 19-21, (2007); http://earthtech.org/hfgw2/.

Landau, L. D. and Lifshitz, E. M., The Classical Theory of Fields, Fourth Revised English Edition, Pergamon Press, (1975), pp. 348, 349, 355-357.

Landy, N. I., Sajuyigbe, S,. Mock, J. J., Smith, D. R. and Padilla, D.R., "Perfect Metamaterial Absorber," Physical Review Letters 100, (2008), pp. 207402-1-4.

Leonhart, U., "Optical Conformal Mapping," Science 312, (2006), pp. 1777-1780.

Li, F. Y., Tang M. and Zhao P., "Interaction Between Narrow Wave Beam-Type High Frequency Gravitational Radiation and Electromagnetic Fields," Acta Physica Sinica 41, (1992), pp. 1919-1928.

Li, F. Y. and Baker, R. M L, Jr., "Detection of High-Frequency Gravitational Waves by Superconductors," 6th International Conference on New Theories, Discoveries and Applications of Superconductors and Related Materials, Sydney, Australia, January 10; Intern. Journ. of Mod. Phys, 21, Nos. 18-19, (2007), pp. 32743278.

Li, F. Y. and Tang, M.X., "Positive definite problem of energy density and radiative energy flux for pulse cylindrical gravitational wave," Acta Physica Sinica 6, (1997), pp. 321-333.

Li,, F. Y., Meng-Xi Tang, Jun Luo, and Yi-Chuan Li , "Electrodynamical response of a high-energy photon flux to a gravitational wave," Phys.l Rev. D 62, , (2000), pp. 044018-1 to 044018 -9.

Li, F. Y., Meng-Xi Tang, and Dong-Ping Shi, "Electromagnetic response of a Gaussian beam to high-frequency relic gravitational waves in quintessential inflationary models," Phys. Rev. B 67, (2003), pp. 104006-1 to -17.

Li, F. Y. and Yang, N., "Resonant Interaction between a Weak Gravitational Wave and a Microwave Beam in the Double Polarized States Through a Static Magnetic Field," Chin. Phys. Lett. 21, No. 11, (2004), p. 2113.

Li, F. Y. and Li, R., "Ultra-High-Intensity Lasers for Gravitational Wave Generation and Detection," in the Proceedings of the Space Technology and Applications International Forum (STAIF-2006), edited by M.S. El-Genk, American Institute of Physics Conference Proceedings 813, Melville New York, (2006), pp.12491258. Available at: http://www.drrobertbaker.com/docs/AIP;\%20HFGW\%20Laser\%20Generator.pdf

Li, F. Y. and Baker, R. M L, Jr. , "Detection of High-Frequency Gravitational Waves by Superconductors," 6th International Conference on New Theories, Discoveries and Applications of Superconductors and Related 
Materials, Sydney, Australia, January 10; International Journal of Modern Physics 21, Nos. 18-19, (2007), pp. 3274-3278.

Li, F.Y., Baker, R. M L , Jr., Fang, Z.. Stephenson, G. V. and Chen, Z., "Perturbative Photon Fluxes Generated by High-Frequency Gravitational Waves and Their Physical Effects," The European Physical Journal C. 56, (2008), pp. 407-423. http://www.drrobertbaker.com/docs/Li-Baker\%206-22-08.pdf

Li. F. Y., Yang, N., Fang. Z., Baker, R. M L, Jr., Stephenson, G. V. and Wen, H., "Signal Photon Flux and Background Noise in a Coupling Electromagnetic Detecting System for High Frequency Gravitational Waves," Phys. Rev. D 80, (2009), pp. 064015-1-13.

Minter, S. J., Wegter-McNelly, K. and Chiao, R. Y., “Do Mirrors for Gravitational Waves Exist?” (2009), arXiv:0903.0661v5, March 24.

Misner, C. W. Thorne, K. and Wheeler, J. A., Gravitation, W. H. Freeman and Company, New York, (1973).

Nishizawa, A., Kawamura, S. Akutsu, T., Arai, K., Yamamoto, K., Tatsumi, D., Nishida, E., Sakagami, M., Takeshi Chiba, Takahashi, R., and Sugiyama, N., "Laser-interferometric detectors for gravitational wave backgrounds at $100 \mathrm{MHz}$ : Detector design and sensitivity," to be published Physical Review D15, (2008).

Pinto, I. M. and Rotoli, G., "Laboratory generation of gravitational waves?" Proceedings of the 8th Italian Conference on General Relativity and Gravitational Physics, Cavlese (Trento), August 30 to September 3, World Scientific-Singapore, (1988), pp. 560-573.

Pendry, J. B., Schurig, D. and Smith, D. R., “Controlling Electromagnetic Fields,” Science 312, (2006), pp. 1790-1782.

Romero-Borja, F. and Dehnen, H., "Generation of gravitational radiation in the laboratory," $Z$. Naturforsch 36a, (1981), pp. 948-955.

Rudenko, V. N. ), "Optimization of parameters of a coupled generator-receiver for a gravitational Hertz experiment," paper HFGW-03-113, Gravitational-Wave Conference, The MITRE Corporation, May 6-9, (2003).

Scully, M. O. and Svidzinsky, A. A. (2009), The Super of Superradiance,” Science 325, pp.1510-1511.

Shawhan, P. S. , "Gravitational Waves and the Effort to Detect them," American Scientist 92, (2004), p. 356.

Stephenson, G. V., "Lessons for Energy Resonance HFGW Detector Designs Learned from Mass Resonance and Interferometric LFGW Detection Schemes," in the Proceedings of the Space, Propulsion and Energy Sciences International Forum (SPESIF), edited by Glen Robertson, American Institute of Physics Conference Proceedings 1103, Melville, New York, (2009a), pp. 532-541. http://www.gravwave.com/docs/Detector\%20Development.pdf

Stephenson, G. V. , "The Standard Quantum Limit for the Li-Baker HFGW Detector," in the Proceedings of the Space, Propulsion and Energy Sciences International Forum (SPESIF), edited by Glen Robertson, American Institute of Physics Conference Proceedings 1103, Melville, New York, (2009b), pp. 542-547. http://www.gravwave.com/docs/HFGW\%20Detector\%20Sensitivity\%20Limit.pdf

Szekeres, P. , "Colliding Plane Gravitational Waves," J. Math. Phys. 13, No. 3, (1972), pp. 286-294.

Woods, R. C. and Baker, R. M L, Jr., "Gravitational Wave Generation and Detection Using Acoustic Resonators and Coupled Resonance Chambers," in the Proceedings of the Space Technology and Applications International Forum (STAIF-2005), edited by M.S. El-Genk, American Institute of Physics Conference Proceedings 746, Melville, New York, (2005), p. 1298.

Woods, R. C., "High-Frequency Gravitational Wave Optics," in the Proceedings of the Space Technology and Applications International Forum (STAIF-2006), edited by M.S. El-Genk, American Institute of Physics Conference Proceedings 813, Melville, New York, (2006), pp. 1305-1312.

Woods, R. C. and Baker, R. M L, Jr., "Generalized Generators of Very-High-Frequency Gravitational Waves Including Ring and Helix Devices," in the Proceedings of the Space, Propulsion and Energy Sciences International Forum (SPESIF), edited by Glen Robertson, American Institute of Physics Conference Proceedings 1103, Melville, New York, (2009), pp. 515-523. 